\title{
Multiverse Spacetime
}

\author{
Khaled (Moh'dKhalaf) Suleiman Alnobani \\ Chemical Engineering, Jordan University of Science and Technology, Irbid 22110, Jordan
}

Received: August 14, 2017 / Accepted: August 22, 2017 / Published: September 30, 2017

\begin{abstract}
A theological train of reasoning is avoided in this paper. The properties of the equal operator presented earlier by Alnobani are given here. A view of the present time is given. As a consequence of the presence of time state, the verses are arranged in couples and every verse including in which we live is a live verse in the same context of human life. This paper interferes with and changes the procedures and philosophy of mathematics itself. Space, mass and time are parts of one whole which is called simply time. The aim of this paper is to make the knowledge of its subject public, so other researchers can contribute to it. The concept of a gate between verses is investigated.
\end{abstract}

Key words: Time, space, multiverse, gate.

\section{Nomenclature}

$\begin{array}{ll}A & \text { Area } \\ B & \text { Magnetic field } \\ C & \text { Constant of integration } \\ c & \text { Speed of light }=2.99792458 \times 10^{8} \mathrm{~m} / \mathrm{s} \\ E & \text { Electrical field } \\ H & \text { Hamiltonian } \\ m & \text { Mass } \\ p & \text { Momentum } \\ q & \text { General coordinate system } \\ s & \text { Length } \\ t & \text { Time } \\ T & \text { Kinetic energy } \\ U & \text { Potential energy field } \\ \theta & \text { Angle } \\ \hbar & \text { Physical constant }=1.0546 \times 10^{-34} \mathrm{~J} \cdot \mathrm{s} \\ \delta & \text { Variation } \\ \varepsilon_{0} & \text { Permittivity of vacuum } \\ \mu_{0} & \text { Permeability of vacuum } \\ \Phi & \text { Flux } \\ = & \text { Mathematical operator }\end{array}$

\section{Subscripts}

$\begin{array}{ll}B & \text { Magnetic } \\ E & \text { Electric } \\ i, j, k & \text { Index } \\ 0 & \text { Initial value }\end{array}$

Corresponding author: Khaled (Moh'dKhalaf) Suleiman Alnobani, M.Sc., research field: chemical engineering.

\section{Introduction}

When someone talks about space the first thing comes to mind is outer space. It is empty and massless. In our context mass occupies a space. Space denotes the property in virtue of which rigid bodies can occupy different positions [1]. This paper is not concerned with elementary particles motion. This work will set the way clear for other researchers to examine the theory and practice of energy and multiverse set forth.

It is hard to believe that neither our current math nor our current physics are correct. Einstein to Lemaitre: "Your math is correct, but your physics is abominable." [2]. Quantum vacuum energies represent an enormous and unobserved dark energy component [3, 4]. Can scientists assure that new discovered elementary particles discovered constantly belong to our verse and not coming from other verses?

\section{Discussion}

\subsection{Equal Operator Properties}

Yet the equal operator [5] has three properties:

(1) Different simultaneous quantities for the same variable could be substituted in the same equation at different sides of the equation. This property was used 
in discovering the time state.

(2) Current quantity of the value is used when certain operations need to use the value in the equation (e.g., $m$ is used instead of $m_{0}$ when $m_{0}$ is squared). Obviously this is not a kind of a known operation. This property was used in the derivation of mass operator.

(3) The imaginary number (square root of -1) is not accepted in the equation.

If we to accept the presence of multi verses, as occupants of this verse must accept the equal operator as it is given herein, the reasoning is that it guarantees the justice and integrity within. Simply this operator is a basis for what is called here "Ethical Mathematics".

One of the applications of the equal operator is in the following equation.

$$
m=m_{0} / \sqrt{1-v^{2} / c^{2}}
$$

To avoid imaginary mass when the velocity is greater than the speed of light an updated speed of light is used in the square of speed of light. The direct reasoning for such a substitution could be found in the curvature of space, on a sphere there are different values for the same parameter (the distance between parallel lines at different points).

\subsection{Present Time Pool}

As the initial state of the whole verses is known and the past is recorded, then the information about the present at any time is known. The future is known only to a supreme knowledge power because of the (choice) dilemma. In the present, the time dimension is perpendicular to the space direction [5]. From an orthogonal point of view all verses appear at the same place, but from the other orthogonal side the time line appears to connect different verses.

If light is a constant, then space and time must be a single unit. Altering the rate of one will alter the rate of the other. In any verse of multiverse light is not constant, then special relativity does not apply (special relativity might be a past knowledge with respect to our current knowledge).

In Fig. 1, every arrow represents time barrier. Time
Connectors' arrows : time

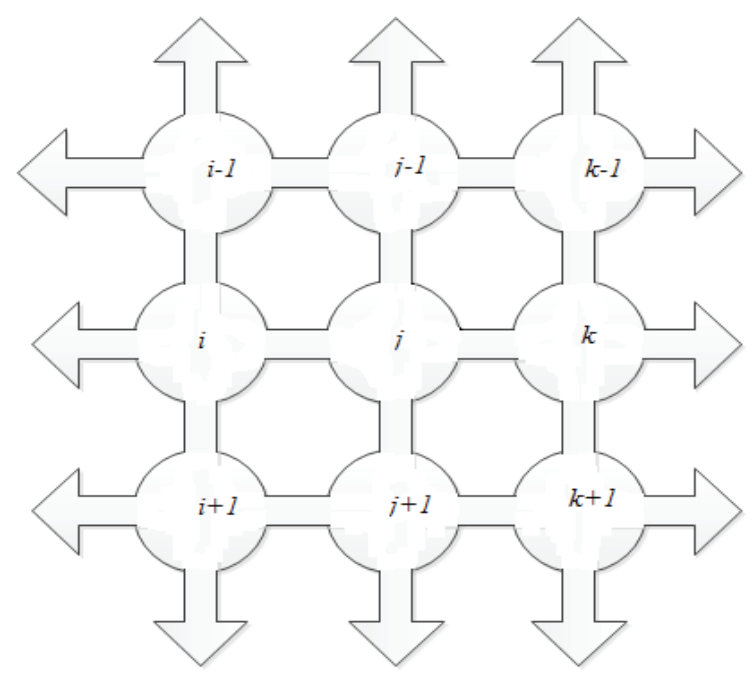

Fig. 1 Verses' matrix.

separates the elements of the verses' matrix and space condenses them (they are all in the same space). Verses are not arranged with respect to their place but with respect to their time. Every orthogonal direction of time corresponds to a different verse which means that the verses shown in Fig. 1 are couples. The space of a universe is a dot product space (Hilbert Space). As a verse behaves as if it was alone in time the combined whole verses are Hilbert space.

From Alnobani [6]:

$$
\overrightarrow{q_{l}} \cdot \overrightarrow{p_{l}}=2 \hbar
$$

This equation governs the space time in a universe.

Divided by the kinetic energy to get time:

$$
\begin{gathered}
\frac{\overrightarrow{q_{l}} \cdot \overrightarrow{p_{l}}}{c^{2}\left(m_{0}-m\right)}=\frac{2 \hbar}{c^{2}\left(m_{0}-m\right)}=t \\
\frac{d m}{d t}=\dot{m}=\frac{2 \hbar}{c^{2} t^{2}}
\end{gathered}
$$

The rate of change of mass is inversely proportional to square of time and to square of length $\left(c^{2} t^{2}\right)$. This shows that mass time rate of change could be expressed both in space and time dimensions. This could be described as the mass breathing of the verse.

The common in verses is that the space of any verse has some kind of coordinate system. And the material (solid time) within has momentum (some kind of 
motion). What is important is that when transferring to other verses would we remain in one piece? It is apparent that the time current flowing in a verse depends on the parameters inside that verse this means that when transferring from verse to verse the rate of change of time may change and when going back to the original verse the span of time is not the same. There are different spans of time for the same change in different verses. There is no independent source to tell us that the electro-magnetic phenomena will hold completely in all verses.

\subsection{Maxwell's Equations}

Maxwell's equations from Ref. [7]:

Gauss's law (electric field):

$$
\oint \vec{E} \cdot d \vec{A}=\frac{(\text { Electric Charge })}{\varepsilon_{0}}
$$

Gauss's law (magnetic field):

$$
\oint \vec{B} \cdot d \vec{A}=0
$$

Faraday's law: $\oint \vec{E} \cdot d \vec{s}=-\frac{d \Phi_{B}}{d t}$

$$
\text { Ampere's law: } \oint \vec{B} \cdot d \vec{s}=\mu_{0} \varepsilon_{0} \frac{d \Phi_{E}}{d t}+\mu_{0} I
$$

From the last two equations it is concluded that rate of change with respect to time is related to line contours not surface contours. The time rate of change is perpendicular to the contour. The object to be transferred (from verse to verse) moves orthogonal to the plane of contour lines' surface (collection of contour lines), what kind of surface could be used as a gate and of what properties? Any surface could be a gate at any time. On the gate one time direction refers to the current verse, the other orthogonal direction refers to other verse (either go through or collision). The gate is as thick as a shadow. Otherwise, it will have dual entity belonging to multiple verses at the same time, with orthogonal time directions such a gate will be a live gate or live creature not only a mere gate. If a key is needed to open a gate then there is a code to be solved, this kind of a gate is previously prepared.

\subsection{Hamilton's Principle}

From Hamilton [8]:

$$
\frac{d p}{d t}=-\frac{\partial H}{\partial q}
$$

and

$$
\frac{d q}{d t}=+\frac{\partial H}{\partial p}
$$

As $t=\frac{q p \cos \theta}{c^{2}\left(m_{0}-m\right)}$,

$$
\begin{aligned}
& q=\frac{t c^{2}\left(m_{0}-m\right)}{p \cos \theta} \\
& p=\frac{t c^{2}\left(m_{0}-m\right)}{q \cos \theta}
\end{aligned}
$$

$\frac{d q}{d t}$

$$
\begin{gathered}
=\frac{c^{2}\left(m_{0}-m\right)}{p \cos \theta} \frac{p \cos \theta(-\dot{m})-c^{2}\left(m_{0}-m\right)(\dot{p} \cos \theta)}{p^{2} \cos ^{2} \theta} \\
=-\frac{c^{2}\left(m_{0}-m\right)\left(p \dot{m}+c^{2}\left(m_{0}-m\right) \dot{p}\right)}{p^{3} \cos ^{2} \theta}=+\frac{\partial H}{\partial p}
\end{gathered}
$$

Similarly,

$$
\begin{gathered}
\frac{d p}{d t}=\frac{c^{2}\left(m_{0}-m\right)}{q \cos \theta}=-\frac{\partial H}{\partial q} \\
H=\frac{c^{2}\left(m-m_{0}\right)}{\cos \theta} \ln q+C .
\end{gathered}
$$

The partial derivative implications could leave the time argument as constant but is it really practical to assume time as constant. Is it practical to take mathematical assumptions as events? In the formulation of this work only practical existence is taken while mathematical implications are not taken unless they really exist. For the total energy to be infinity, time must not pass and it must remain as, not to go to future or past. Present must remain present, not moving forward or backward. This involves in the heart of the differentiation process.

\subsection{Time Change}

If there was not electromagnetic phenomena, does time proceed? Are other verses collected together by the same forces of nature as ours? These questions concern our material and other verses' material. These 
kinds of questions should be asked if to transfer from verse to verse. Change of verse is merely change of time, which is a change in material itself. However, an outside (other than human deductive reasoning, might be religious ${ }^{1}$ ) hint to decide about the suitability of other verses for human life is needed.

\section{Results}

As the two coupled verses have orthogonal time directions and as their dot product equals zero (vacuum) then from Ref. [6] these two verses are alive. The possible existing verses are live verses in the same context as human life.

We should stop looking to time as an imaginary axis, because if we are to control it, it should be real around us and could be obviously seen.

The mass of the transferring object (from verse to another verse) should be twice its initial mass (by creation of Eq. (1)) so that conservation of mass in the original verse will hold. When transferring, the initial mass transfer, while the rest creation remains in some form in the original verse. From mass relativity equation (Eq. (1)) the velocity possible for the transferring object could be found. As life is time void (vacuum) its transfer does not need restrictions on mass or velocity.

From Eq. (1):

$$
v=\frac{\sqrt{3}}{2} c=2.596278845 \times 10^{8} \mathrm{~m} / \mathrm{s}
$$

Recall that: $\cos 30^{\circ}=\frac{\sqrt{3}}{2}$.

This is the actual velocity of transfer, it is not a restriction, but it is a requirement for mass conservation to pass the gate with absolute verse frame of reference. This is the velocity within the gate not the velocity to approach the gate.

From the Hamiltonian equation (Fig. 2) previously

\footnotetext{
${ }^{1}$ Quran, 21, The Prophets -Al-'Anbiya, [21.30] "Have the unbelievers not seen that the heavens and the earth were sewn up as one (solid) mass, then We unstitched them, and that We made every living thing of water? Will they not believe!" All verses to be known to exist for us contain what we know as water (because it is alive), all have the same forces of nature.
}

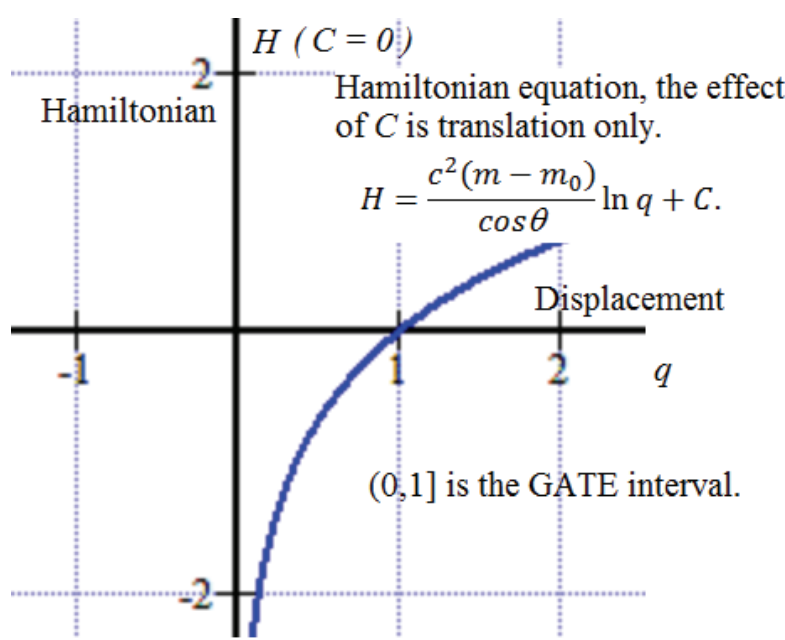

Fig. 2 Hamiltonian equation.

derived the total energy is not defined at zero distance from the object.

The object is always subject to an open gate if its energy reaches $-\infty$. The gate path extends from zero distance (zero is not included) to 1. Minus infinity energy could be achieved in more than one way:

(1) Zero mass (vacuum or living creature). Any living creature (time vacuum) is subject to transfer - or be open - to another verse at any time (without its accompanying mass).

(2) Reaching $-\infty$ energy.

(3) The angle between momentum and its resultant distance is $\pi / 2$. This requires violation of the conservation of momentum principle. Also, it requires that the object is decelerating (annihilation).

Things could be seen as follows: The conservation principles (conservation of energy, conservation of linear momentum, conservation of angular momentum, and conservation of electric charge) are the guarantee of remaining in the same verse.

The time required to pass the path of the gate is $3.851666403 \times 10^{-9} \mathrm{~s}$, or

$$
\mathrm{t}=\frac{2}{\sqrt{3}} \frac{1}{\mathrm{c}}
$$

if our units of measurement are assumed to be valid. This time is required to transfer to other verses or to transfer to any place (irrespective of distance) in the same verse (if the gate returns the object transferred to the same original verse). The reciprocal of this time is 
the number of verses that could be transferred to in one second (with the requirement of $-\infty$ energy). If the gate is open and the transfer happens it could not be used to go back, another gate should be opened. The gate is not a plane to go through, it is a condition when the object violates the conservation principles. If a plane-like-gate is used, the system transferred would disintegrate (even if transferring to the same verse) because a part of it would transfer while the rest remains in the original verse.

\section{Conclusions}

There is no limit for exchange of mass between verses. Consequently there is no limit for annihilation and creation processes. The "Black Hole" is not seen here as a gate, it is seen as a compressor (compress matter). The objects gone to a black hole will be compressed within; not going to any other world. Otherwise, our verse will be seen from other worlds by the material taken from it and it will be uncovered and crowded by other world's occupants which are not the case. Opening a gate is a process that involves more than one verse. It is simultaneously working on more than one verse. The dilemma is not opening a gate in the current verse, it is the other verse that counts most. There are many possible gates and it must be decided which gate to open. To open a gate it is needed to expand (or contract) time within space to the new verse setting (time space). Where this is most likely to happen? Around massive objects and living beings (vacuum). For the fact that living beings are subject to open their conscious to multiple verses, the phases of psychiatric theories and consequently therapy should change.

\section{References}

[1] Encyclopedia Britannica Retrieved 10 April.

[2] Deprit, A. 1984. "Monsignor Georges Lemaître." In The Big Bang and Georges Lemaître, edited by A. Barger. Reidel, 370.

[3] Zel'dovich, Y. B. 1967. "Cosmological Constant and Elementary Particles.” JETP Letters 6 (9): 316-7.

[4] Zel'dovich, Y. B. 1968. "The Cosmological Constant and the Theory of Elementary Particles." Soviet Physics Uspekhi 11 (3): 381-93.

[5] Hermann, M. 1909. "Raum und Zeit." Physikalische Zeitschrift 10 (18): 75-88.

[6] Alnobani, K. S. 2017. "Multiverse and the Time State." Journal of Energy and Power Engineering 11 (3): 160-3.

[7] Fleisch, D. 2008. A Student's Guide to Maxwell's Equations. Cambridge University Press.

[8] Hamilton, W. R. 2000. "On a General Method in Dynamics." In Philosophical Transaction of the Royal Society, Part II (1834): 247-308; Part I (1835): 95-144. From the collection Sir William Rowan Hamilton (1805-1865): Mathematical Papers edited by David R. Wilkins, School of Mathematics, Trinity College, Dublin 2, Ireland. 